Published in final edited form as:

Nat Biotechnol. 2009 March ; 27(3): 275-280. doi:10.1038/nbt.1529.

\title{
Highly efficient neural conversion of human ES and iPS cells by dual inhibition of SMAD signaling
}

\author{
Stuart M. Chambers ${ }^{1}$, Christopher A. Fasano ${ }^{1}$, Eirini P. Papapetrou ${ }^{2}$, Mark Tomishima ${ }^{1}$, \\ Michel Sadelain ${ }^{2}$, and Lorenz Studer ${ }^{1,3}$ \\ ${ }^{1}$ Developmental Biology Program, Sloan-Kettering Institute, 1275 York Ave, New York, New York \\ 10065, USA. \\ ${ }^{2}$ Center for Cell Engineering, Memorial Sloan-Kettering Cancer Center, New York, New York, \\ USA
}

${ }^{3}$ Department of Neurosurgery, Sloan-Kettering Institute, 1275 York Ave, New York, New York 10065, USA.

\begin{abstract}
Current neural induction protocols in human ES cells (hESCs) rely on embryoid body formation, stromal feeder co-culture, or selective survival conditions; each strategy displaying significant drawbacks such as poorly defined culture conditions, protracted differentiation and low yield.

Here we report that the synergistic action of two inhibitors of SMAD signaling, Noggin and SB431542, is sufficient for inducing rapid and complete neural conversion of hESCs under adherent culture conditions. Temporal fate analysis reveals a transient $\mathrm{FGF}^{+}$epiblast-like stage followed by $\mathrm{PAX}^{+}$neural cells competent of rosette formation. Initial cell density determines the ratio of CNS versus neural crest progeny. Directed differentiation of human iPSCs into midbrain dopamine and spinal motoneurons confirm robustness and general applicability of the novel induction protocol.
\end{abstract}

Noggin/SB431542 based neural induction should greatly facilitate the use of hESC and hiPSCs in regenerative medicine and disease modeling and obviate the need for stromal feeder or embryoid body based protocols.

Human ESCs offer great promise for cell-replacement therapies, and recent advances in somatic cell reprogramming to induced pluripotent stem cells (iPSCs) has opened the door to generating patient-specific cells for regenerative medicine and disease modeling1.

However, to realize the full potential of these approaches, improved differentiation protocols are required that eliminate the use of undefined factors such as neural-inducing stroma (PA6 or MS5 cells2, 3), the heterogeneous nature of embryoid body differentiation or the poor

Users may view, print, copy, and download text and data-mine the content in such documents, for the purposes of academic research, subject always to the full Conditions of use:http://www.nature.com/authors/editorial_policies/license.html\#terms

Correspondence: Dr. Lorenz Studer, Developmental Biology, 1275 York Ave, Box 256, New York, NY 10065, Phone: 212-639-6126, Fax: 212-717-3642, Email: studerl@mskcc.org.

Author Contributions

S.M.C. and L.S. designed the study. E.P.P., M.T., L.S., and M.S. designed and generated the h-iPS clones. S.M.C. and L.S. analyzed the data and wrote the manuscript. S.M.C. and C.A.F. performed the experiments. 
yield of protocols based on selective survival of neural progeny. Understanding and triggering the signaling pathways necessary and sufficient for neural induction in hESCs is a critical goal in this effort.

Several lines of evidence demonstrate a crucial role for SMAD signaling during neural induction. Elegant studies in frog identified BMP inhibitors including chordin4, follistatin5, and noggin6 as the critical neural inducing factors in the Speamann organizer. Mammalian noggin7 has comparable neural inducing properties, and treatment with recombinant Noggin has been used in several hESC neural induction protocols3, 8. More recently, the drug SB431542 was shown to enhance neural induction in an embryoid body (EB) based hESC neural induction protocol9. SB431542 inhibits the Lefty/Activin/TGF $\beta$ pathways by blocking phosphorylation of ALK4, ALK5, ALK7 receptors. While Noggin or SB431542 treatment improve the efficiency of neural induction, neither treatment alone is sufficient to neurally convert hESCs under defined or adherent conditions. Here we set out to test whether combined blockade of SMAD signaling using Noggin and SB431542 is sufficient to achieve full neural conversion and to obviate the need for EB- or stromal-feeder based protocols.

We postulated that establishing an even cell distribution is critical for inducing homogenous neural differentiation of hESCs. Therefore, undifferentiated hESC were dissociated into single cells and re-plated onto matrigel coated dished in conditioned medium supplemented with the ROCK-inhibitor, Y-2763210, promoting survival of hESC as single cells (for details see M\&M). After 72 hours cells were switched from hESC conditions to knock-out serum replacement media (KSR) containing either Noggin, SB431542, or both factors and allowed to differentiate for a total of 11 days (Fig. 1a). The greatest reduction in nuclear localization of the obligate co-SMAD, SMAD4, was observed after 24 hours when both Noggin and SB431542 were present (Supplementary Figure 1). Neural induction was monitored by expression of PAX6, an early marker of neurectodermal differentiation 11 . Combined treatment with Noggin and SB431542 dramatically increased the efficiency of neural induction to greater than $80 \%$ of total cells, compared with less than $10 \%$ PAX6 $^{+}$ cells when Noggin or SB431542 were used alone (Fig. 1b). There are several potential mechanisms that could contribute to the synergistic action of Noggin and SB431542. These include destabilizing the activin- and Nanog-mediated pluripotency network12, suppression of BMP induced differentiation towards trophoblast lineage13, suppression of mes-/ endodermal fates by inhibiting endogenous activin and BMP signals14, 15 and promoting neuralization of primitive ectoderm by BMP inhibition16 (Supplementary Figure 2). Temporal analysis of gene expression revealed treatment with SB431542 induced a rapid loss of Nanog expression (Supplementary Figure 3) and a dramatic increase in the expression of CDX2 (Fig. 1c). These data suggest SB431542 mediated loss of pluripotency is associated with differentiation towards trophoblast lineage. Suppression of CDX2 in the presence of Noggin or Noggin/SB431542 demonstrates that one key roles of Noggin is the repression of endogenous BMP signals that drive trophoblast fates upon differentiation. The pronounced induction of SOX1 in Noggin/SB431542 treated cultures confirmed a strong bias towards neurectodermal lineage in the dual SMAD inhibition protocol. There is also evidence for suppression of alternative embryonic germ layers such as Noggin-mediated suppression of SOX17 (endodermal lineage) and SB431542-mediated suppression of 
Brachyury (mesodermal lineage) (Fig. 1c). Taken together, these results indicate that SB431542 and Noggin work synergistically at multiple stages of differentiation to achieve efficient neural conversion of hESCs.

We next sought to characterize lineage progression of hESC progeny after the addition of the two inhibitors. Immunocytochemical analysis showed loss of OCT4 expression by day 5 and strong expression of Pax 6 by day 7 (Fig. 1d). These data pointed to the presence of an intermediate cell type at day 5 of differentiation that was negative for both OCT4 and PAX6. Gene expression analysis revealed peak expression of the epiblast marker FGF5 at day 5 of differentiation concomitant with high expression of OTX2, another epiblast marker whose expression is maintained during neural fate commitment (Fig. 1e). The expression of OTX2 and FGF5 prior to neuralization suggests the presence of a human epiblast stage cells in our cultures17. Interestingly, the earliest neural marker expressed in our culture system was SOX1 (Fig. 1f), preceding induction of other neurepithelial markers such as ZIC1 or PAX6, and preceding expression of anterior CNS (FOXG1) and neural crest (p75) markers. While previous studies had suggested PAX6 preceded SOX1 expression18, early induction of SOX1 has been observed in mouse cultures19. One interesting possibility to explain early SOX1 expression could be direct modulation of SOX1 transcription by SMAD signaling in our culture system.

Our lab has recently described methods for establishing stable mouse20 and hESC21 transgenic reporter lines carrying bacterial artificial chromosomes (BACs) engineered to express GFP under control of cell type specific promoters. Here we used the HES5: :eGFP BAC transgenic hESC reporter line, marking neural stem and precursor cell progeny 20 (Placantonakis et al.,2008; in press), to measure the efficiency of neural induction. The dual SMAD inhibition protocol was compared to the standard MS5 protocol in the presence of Noggin22. To this end HES5: :eGFP cells were plated in media supplemented with Noggin either in the presence of MS5 feeder cells or SB431542 and allowed to differentiate for 13 days, a stage when the $\mathrm{GFP}^{+}$cells were readily observed under both conditions (Supplementary Figure 4). GFP expression was quantified by flow cytometry. Non-modified H9 cells were used as negative controls. MS5 cells were excluded from the analysis based on negative selection for the cell surface molecule CD105 (Supplementary Figure 5). Dual SMAD inhibition yielded $82 \% \mathrm{GFP}^{+}$cells at day 13 , a more than 3 fold increase compared with the MS5/Noggin protocol (Fig. 1f). In contrast to the MS5 protocol which requires plating of hESC colonies at low density in the presence of a MS5 mouse stromal cell line22, the Noggin/SB431542 condition allowed for high plating densities. Therefore, in addition to higher percentages, the dual SMAD inhibition protocol also resulted in larger absolute numbers of Hes5: :eGFP+ cells per each culture plate (data not shown).

We previously reported the isolation of rosette neural stem cells8 (R-NSCs) and neural crest stem cells3 (NCSCs) from hESCs. We next sought to determine the lineage relationship of the early $\mathrm{PAX6}^{+}$neurectodermal cells observed in the dual SMAD inhibition protocol to the R-NSCs and NCSCs populations described previously. Immunocytochemical analysis showed that, similar to R-NSCs, PAX6 ${ }^{+}$neurectodermal cells express general NSC markers such as Nestin and R-NSC markers including promyelocytic leukemia zinc finger (PLZF; Fig. 2a,b; day 11 of differentiation). However, cytoarchitecture and ZO1 expression 
indicated that neuroepithelial cells under these conditions were non-polarized exhibiting a more primitive ESC-like cytoarchitecture. These non-polarized areas were interspersed with R-NSC like areas composed of polarized columnar epithelial cells (Fig. 2c). The developmental hierarchy of these two cell populations was further explored upon subsequent passage. Early neuroepithelial cells spontaneously converted into rosette structures with apical ZO1 expression and evidence of interkinetic nuclear migration post-passage (Fig. 2d). These data suggest that the Noggin / SB431542 protocol yields an early PAX6 ${ }^{+}$ neuroepithelial population capable of rosette formation. The early $\mathrm{PAX}^{+}$cells may therefore represent the most primitive hESC derived neural precursor stage isolated to date. R-NSCs have been shown to acquire anterior CNS marker by default8.

$\mathrm{PAX}^{+}$neuroepithelial cells generated via the dual SMAD inhibition protocol exhibited an anterior CNS character as evidenced by expression of Otx2 and FoxG1B (Fig. 2e,f) similar to R-NSCs8. Interestingly, PAX6 negative cells under these conditions co-expressed markers of neural crest including AP2, HNK1, PAX7, and p75 (NGFR) (Fig. 2g-j). Manipulations of the initial hESC plating density skewed the ratio of PAX6 ${ }^{+} \mathrm{CNS}$ versus $\mathrm{PAX6}^{-}$neural crest-like cells. High plating densities resulted in near exclusive differentiation towards $\mathrm{PAX}^{+}$cells while low densities promoted neural crest-like differentiation (Supplementary Figure 6). The presence of large numbers of neural crest-like cells prior to rosette formation suggested that dual SMAD inhibition yields an early neural crest population distinct from R-NSC derived NCSCs3. Supporting the notion of an early neural crest population with distinct lineage potential cells could be readily enriched for pigmented cells co-expresssing the melanosome marker, HMB45 (Fig. 2k,1, see M\&M for details). In contrast, R-NSC derived NCSCs typically do not yield pigmented cells under comparable conditions3. However, not all $\mathrm{HMB} 45^{+}$cells co-expressed the neural crest marker SOX10 suggesting the presence of other pigmented cell populations including retinal pigment epithelial cells arising from the PAX6+ domain (data not shown). It will be interesting in the future to compare differential fate potential of early versus R-NSC derived neural crest precursors in our hESC system with the fate potential of different neural crest precursor populations in vivo.

Anterior-posterior and dorso-ventral identity and neuronal subtype potential is dependent on early exposure to morphogenic factors such as retinoic acid, FGF8, and SHH. We next explored the patterning potential of cells generated via the dual SMAD inhibition protocol. We postulated that day 5 of differentiation may present an appropriate developmental window for neural patterning since Oct 4 expression is silenced between day 3 and 5 and the neural marker PAX6 is activated in the majority of cells between day 5 and 7 (Figure 1d,e).

Derivation of cells expressing markers of dopamine neurons was observed following exposure to SHH and FGF822 starting at day 5 and day 9 of differentiation respectively (Fig. 2m). One week after SHH exposure, both FGF8 and SHH were withdrawn and further differentiated in medium containing BDNF, ascorbic acid, GDNF, TGF- $\beta 3$, and cyclic-AMP (BAGTC22, see Fig. 2m). At day 19 of differentiation neurons a large proportion of Tuj $1^{+}$ neurons co-expressed tyrosine hydroxylase (TH) (Figure 2n,o), the rate-limiting enzyme in the synthesis of dopamine. $\mathrm{TH}^{+}$neurons emerged under these conditions spontaneously even in the absence of cell passaging. However, derivation of more mature $\mathrm{TH}^{+}$cells with long 
neural processes was promoted following mechanical isolation and en bloc passage at day 12 of differentiation.

Nuclear expression of the motoneuron markers ISL1 and HB9 was observed two weeks upon exposure to BDNF, ascorbic acid, SHH, and retinoic acid (BASR; day 19 of differentiation) confirming the derivation of somatic type motoneurons (Fig. 2q,r). Motoneuron derivation was limited to cultures passaged at about day 11 of differentiation (data not shown) suggesting reduced patterning response at very high cell densities as observed for hESC derived R-NSCs8. These data demonstrate a robust patterning response in Noggin/SB431542 treated neural progeny and derivation of relevant neuron subtypes after short differentiation periods ( $\sim 19$ days) compared to $30-50$ days when using stromal feeder mediated induction protocols3, 22 .

Recent publications have reported the reprogramming of human somatic cells into induced pluripotency stem cells (hiPSCs) 1, 23, 24. We wanted to next determine if dual SMAD inhibition could be used to reliably generate a broad repertoire of hiPSC derived neural cell types. Given the expected intrinsic variability among hiPSC clones, reproducible differentiation results would confirm the robustness of our novel differentiation protocol. Two hiPS clones (iPS ${ }^{\mathrm{C} 14}$, iPS ${ }^{\mathrm{C} 27}$; Fig. 3a-i,a-ii) were generated using lentiviral transduction of human fetal lung fibroblasts with cMYC, KLF4, OCT4, and SOX2. Both clones express the pluripotency markers including Nanog, Tra-1-60, and SSEA-3 at the undifferentiated state and are capable of differentiating into derivatives of the three germ layers (data not shown). Upon neural induction via the Noggin/SB431542 protocol, both clones yielded nearly homogenous populations of PAX6 $6^{+}$cells by day 11 of differentiation (Fig. 3b-i,b-ii). Using the strategies described above manipulating, cell density, passage, and patterning factors both hiPSC clones could be readily biased towards generating $\mathrm{HNK}^{+}$ putative neural crest progeny (Fig c-i,c-ii), hiPSC derived R-NSCs (Fig. 3d-i,d-ii), and specific hiPSC derived neuron subtypes including somatic motoneurons (Fig. 3e-i,e-ii) and dopamine neurons (Fig. 3f-i,f-ii). These data demonstrate robustness and modularity of the dual SMAD inhibition strategy beyond hESC differentiation. The novel protocol offers an efficient, defined, and robust platform for the rapid generation of hiPSC derived neural cell types.

In this report, we describe a novel method of neural differentiation that combines the inhibitors SB431542 and Noggin to block SMAD signaling. While for most of the studies presented here an 11-day treatment period was used, preliminary studies indicate that comparable levels of neural induction can be achieved when the treatment is shortened to the first 5 days of differentiation (Supplementary Figure 7; and data not shown). This should further reduce complexity and cost, particularly in the case of recombinant Noggin. SB431542/Noggin treatment greatly improves on current methods of generating neural tissue by inducing rapid and uniform neural conversion of human pluripotent cells under adherent culture conditions without the need for embryoid body formation or MS5 stromal feeder co-culture. The novel protocol allows for the derivation of relevant neuron subtypes after much shorter differentiation periods ( $\sim 19$ days) compared to $30-50$ days of differentiation when using stromal feeder mediated induction protocols3, 22. Given the need for defined protocols that induce rapid and complete neural conversion, we predict that this 
technique may become the standard strategy for driving differentiation in human pluripotent cells.

\section{Materials and Methods Cells and culture conditions}

hESC (WA-09; passages 35-45) and IPS lines (iPS ${ }^{\mathrm{C14}}$, iPS ${ }^{\mathrm{C} 27}$; passages 4-10) were cultured on mouse embryonic fibroblasts plated at $12-15,000 \mathrm{cells} / \mathrm{cm}^{2}$ (MEFs, Globalstem). A medium of DMEM/F12, 20\% knock-out serum replacement (Gibco), 0.1 $\mathrm{mM}$ b-mercaptoethanol, $6 \mathrm{ng} / \mathrm{mL}$ FGF-2 was changed daily. Cells were passaged using 6 $\mathrm{U} / \mathrm{mL}$ of dispase in hESC media, washed and re-plated at a dilution of 1:5 to 1:10.

\section{IPS cell generation}

The cDNAs encoding hOct4, hSox2, hKlf4 and c-myc (purchased from Open Biosystems) were subcloned into self-inactivating lentiviral vectors driven by the human phosphoglycerate kinase (PGK) promoter. Lentiviral vector supernatants were produced by triple co-transfection of the plasmid DNA encoding the vector, pCMV $\Delta$ R8.91 and pUCMD.G into 293T cells. Human fetal lung fibroblasts (MRC-5) purchased from ATCC (CCL-171) were seeded at $1.5 \times 10^{4}$ cells $/ \mathrm{cm}^{2}$ in Eagle's Minimum Essential Medium supplemented with $10 \%$ fetal bovine serum (FBS). The following day the fibroblasts were transduced with equal amounts of supernatants of the four lentiviral vectors in the presence of $4 \mathrm{ug} / \mathrm{ml}$ polybrene for $\sim 16$ hours. Six days after transduction, fibroblasts were harvested by trypsinization and plated at $2 \times 10^{4}$ cells per $60 \mathrm{~mm}$ dish on a feeder layer of mytomycin C-treated mouse embryonic fibroblasts (CF-1). The next day, the medium was switched to $\mathrm{hESC}$ medium. The iPS lines were confirmed positive for Tra-1-81, Tra-1-60, SSEA-4 and Nanog by immunofluorescence and flow cytometry. In both hiPSC clones all 4 vectorencoded transgenes were found to be silenced.

\section{Neural Induction}

hESC cultures were disaggregated using accutase for 20 minutes, washed using hESC media and pre-plated on gelatin for 1 hour at $37^{\circ} \mathrm{C}$ in the presence of ROCK inhibitor to remove MEFs. The nonadherent hESC were washed and plated on matrigel at a density of 10,00025,000 cells $/ \mathrm{cm}^{2}$ on matrigel (BD) coated dishes in MEF conditioned hESC media (CM) spiked with $10 \mathrm{ng} / \mathrm{mL}$ of FGF-2 and ROCK-inhibitor. Ideal cell density was found to be 18,000 cells $/ \mathrm{cm}^{2}$. The ROCK inhibitor was withdrawn, and hESC were allowed to expand in $\mathrm{CM}$ for 3 days or until they were nearly confluent. The initial differentiation media conditions included knock out serum replacement (KSR) media with $10 \mathrm{nM}$ TGF-b inhibitor (SB431542, Tocris) and $500 \mathrm{ng} / \mathrm{mL}$ of Noggin (R\&D). Upon day 5 of differentiation, the TGF-b inhibitor was withdrawn and increasing amounts of N2 media $(25 \%, 50 \%, 75 \%)$ was added to the KSR media every two days while maintaining $500 \mathrm{ng} / \mathrm{mL}$ of Noggin. For MS5 induction, established methods previously reported were used22.

\section{Quantitative Real-time PCR}

Total RNA was extracted using an RNeasy kit (Qiagen). For each sample, 1 ug of total RNA was treated for DNA contamination and reverse transcribed using the Quantitect RT kit 
(Qiagen). Amplified material was detected using Quantitect SYBR green probes and PCR kit (Qiagen) on a Mastercycler RealPlex2 (Eppendorf). All results were normalized to a HPRT control and are from 4-6 technical replicates of 2-3 independent biological samples at each data point.

\section{Neuronal patterning and differentiation}

Dopaminergic patterning was initiated using BDNF, ascorbic acid, sonic hedgehog, and FGF8 in N2 media as previously reported22, and maturation was performed in the presence of BDNF, ascorbic acid, GDNF, TGFb-1, and cyclic-AMP. Motoneuron patterning was performed using BDNF, ascorbic acid, sonic hedgehog, and retinoic acid in N2 media as previously reported 18 .

\section{Microscopy, antibodies, and flow cytometery}

Tissue was fixed using 4\% paraformaldehyde for 20 minutes, washed with PBS, permeablized using .5\% Triton X in PBS, and blocked using 1\% BSA in PBS. Primary antibodies used for microscopy included PAX6 (Covance), Oct4 (Biovision), AP2 (Novus Biologicals), GBX2 (Sigma), HNK1 (Sigma), HOXB4 (Developmental Studies Hybridoma Bank (DSHB)), Nestin (R\&D), NKX6.1 (DSHB), OTX2 (gift), p75 (Advanced Target Systems.), PAX7 (DSHB), PLZF (Calbiochem), TUJ1 (Covance), ZO1 (Zymed), BF1 (FOXG1, gift Esseng Lai), TH (Sigma), HB9 (DSHB), ISL1 (DSHB). CD105-PE (eBioscience) was used for excluding MS5 stromal cells for flow cytometery on a FACScan (BD).

\section{Supplementary Material}

Refer to Web version on PubMed Central for supplementary material.

\section{Acknowledgements}

We are grateful to F. Vaccarino for providing Otx2 antibody and E. Lai for BF1 antibody. This work was supported in part by the Starr Foundation, NINDS grant 1R01NS052671, the Starr Stem Scholar fellowship (S.M.C.) and the a New York Stem Cell Foundation fellowship (C.A.F.)

\section{References}

1. Takahashi K, et al. Induction of pluripotent stem cells from adult human fibroblasts by defined factors. Cell. 2007; 131:861-872. [PubMed: 18035408]

2. Kawasaki H, et al. Induction of midbrain dopaminergic neurons from ES cells by stromal cellderived inducing activity. Neuron. 2000; 28:31-40. [PubMed: 11086981]

3. Lee H, et al. Directed differentiation and transplantation of human embryonic stem cell-derived motoneurons. Stem Cells. 2007; 25:1931-1939. [PubMed: 17478583]

4. Sasai Y, et al. Xenopus chordin: a novel dorsalizing factor activated by organizer-specific homeobox genes. Cell. 1994; 79:779-790. [PubMed: 8001117]

5. Hemmati-Brivanlou A, Kelly OG, Melton DA. Follistatin, an antagonist of activin, is expressed in the Spemann organizer and displays direct neuralizing activity. Cell. 1994; 77:283-295. [PubMed: 8168135]

6. Smith WC, Harland RM. Expression cloning of noggin, a new dorsalizing factor localized to the Spemann organizer in Xenopus embryos. Cell. 1992; 70:829-840. [PubMed: 1339313] 
7. Valenzuela DM, et al. Identification of mammalian noggin and its expression in the adult nervous system. J Neurosci. 1995; 15:6077-6084. [PubMed: 7666191]

8. Elkabetz Y, et al. Human ES cell-derived neural rosettes reveal a functionally distinct early neural stem cell stage. Genes Dev. 2008; 22:152-165. [PubMed: 18198334]

9. Smith JR, et al. Inhibition of Activin/Nodal signaling promotes specification of human embryonic stem cells into neuroectoderm. Dev Biol. 2008; 313:107-117. [PubMed: 18022151]

10. Watanabe K, et al. A ROCK inhibitor permits survival of dissociated human embryonic stem cells. Nat Biotechnol. 2007; 25:681-686. [PubMed: 17529971]

11. Callaerts P, Halder G, Gehring WJ. PAX-6 in development and evolution. Annu Rev Neurosci. 1997; 20:483-532. [PubMed: 9056723]

12. $\mathrm{Xu} \mathrm{RH}$, et al. NANOG is a direct target of TGFbeta/activin-mediated SMAD signaling in human ESCs. Cell Stem Cell. 2008; 3:196-206. [PubMed: 18682241]

13. Xu RH, et al. BMP4 initiates human embryonic stem cell differentiation to trophoblast. Nat Biotechnol. 2002; 20:1261-1264. [PubMed: 12426580]

14. D'Amour KA, et al. Efficient differentiation of human embryonic stem cells to definitive endoderm. Nat Biotechnol. 2005; 23:1534-1541. [PubMed: 16258519]

15. Laflamme MA, et al. Cardiomyocytes derived from human embryonic stem cells in pro-survival factors enhance function of infarcted rat hearts. Nat Biotechnol. 2007; 25:1015-1024. [PubMed: 17721512]

16. Munoz-Sanjuan I, Brivanlou AH. Neural induction, the default model and embryonic stem cells. Nat Rev Neurosci. 2002; 3:271-280. [PubMed: 11967557]

17. Tesar PJ, et al. New cell lines from mouse epiblast share defining features with human embryonic stem cells. Nature. 2007; 448:196-199. [PubMed: 17597760]

18. Li XJ, et al. Specification of motoneurons from human embryonic stem cells. Nat Biotechnol. 2005; 23:215-221. [PubMed: 15685164]

19. Suter DM, Tirefort D, Julien S, Krause KH. A Sox1 to Pax6 switch drives neuroectoderm to radial glia progression during differentiation of mouse embryonic stem cells. Stem Cells. 2008

20. Tomishima MJ, Hadjantonakis AK, Gong S, Studer L. Production of green fluorescent protein transgenic embryonic stem cells using the GENSAT bacterial artificial chromosome library. Stem Cells. 2007; 25:39-45. [PubMed: 16990587]

21. Placantonakis DG, et al. Bac Transgenesis in Human Es Cells as a Novel Tool to Define the Human Neural Lineage. Stem Cells. 2008

22. Perrier AL, et al. Derivation of midbrain dopamine neurons from human embryonic stem cells. Proc Natl Acad Sci U S A. 2004; 101:12543-12548. [PubMed: 15310843]

23. Yu J, et al. Induced pluripotent stem cell lines derived from human somatic cells. Science. 2007; 318:1917-1920. [PubMed: 18029452]

24. Park IH, et al. Reprogramming of human somatic cells to pluripotency with defined factors. Nature. 2008; 451:141-146. [PubMed: 18157115] 

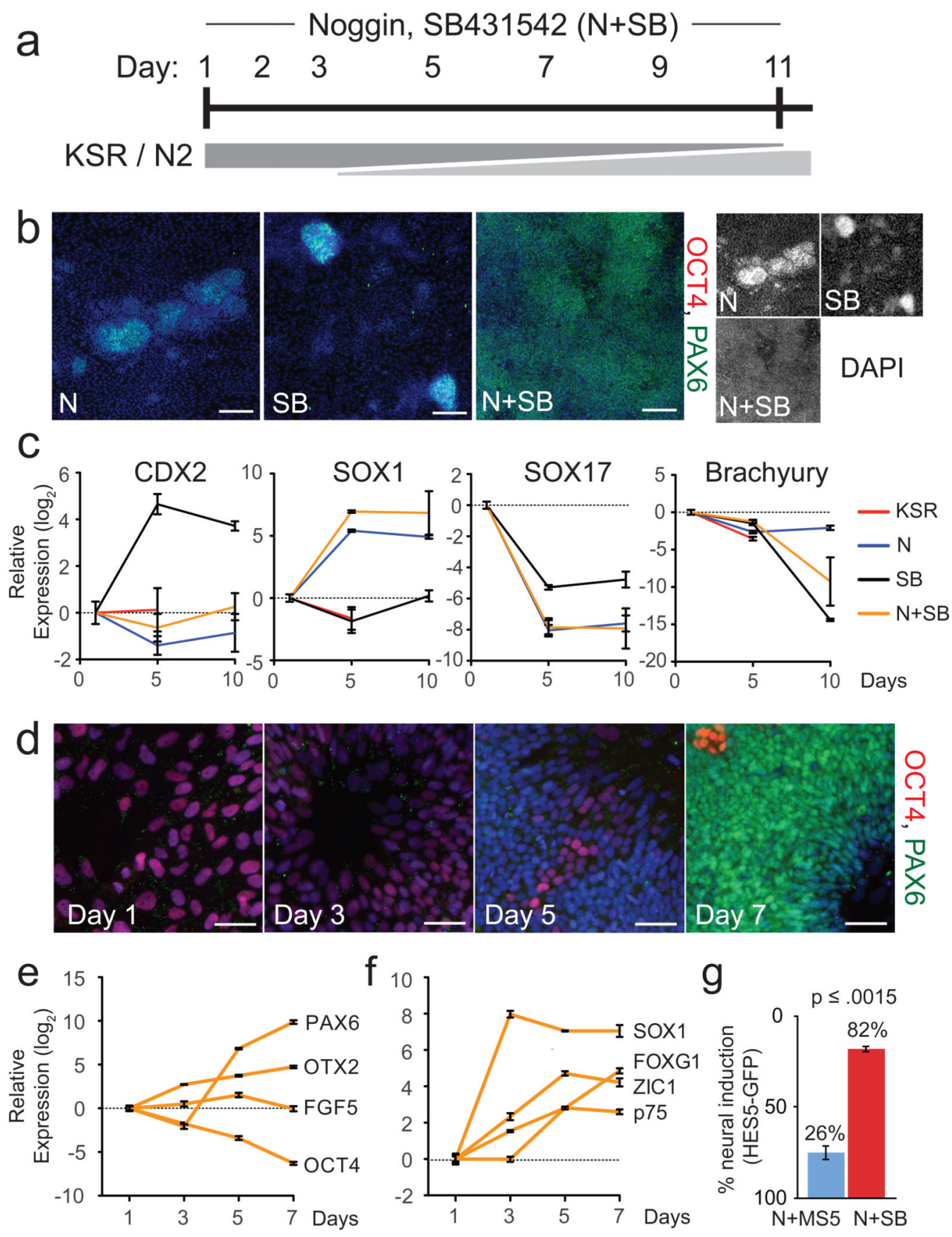

Figure 1.

Dual SMAD inhibition allows for a highly efficient feeder-free neural induction in adherent cultures in seven days. (a) Differentiation scheme used for achieving neural induction can be achieved with the combination of SB431542, an ALK inhibitor, and Noggin, a BMP inhibitor. (b) The dual SMAD inhibition greatly improves neural differentiation (PAX6 expression, green) to greater than $80 \%$. Infrequent neural differentiation $\left(<10 \% \mathrm{PAX}^{+}\right.$ cells) can be observed when the single factors are used. (c) Real-Time PCR for early germ layer markers CDX2, SOX1, SOX17 and Brachyury. (d) Immunoflouresence for OCT4 
(red) and PAX6 (green) expression indicates rapid neutralization occurs by day 7. (e) RealTime PCR for PAX6, OTX2, FGF5, OCT4 during dual SMAD inhibition reveals an epistem cell intermediate at day 5. (f) Real-Time PCR for neural and neuronal markers during dual SMAD inhibition differentiation towards neurectoderm. (g) A BAC reporter line (HES5-GFP) was used to quantify the percentage of neural induction for the method using MS5 stromal cells (with Noggin) or dual SMAD inhibition (SB431542 and Noggin). All error bars represent S.E.M. and the p-value was determined using Student's T-test. Abbreviations: N, Noggin; SB, SB431542; KSR, knock-out serum replacement medium; N2, N2 medium. Scale bars: (b) $-200 \mu \mathrm{m}$; (d) $-50 \mu \mathrm{m}$. 

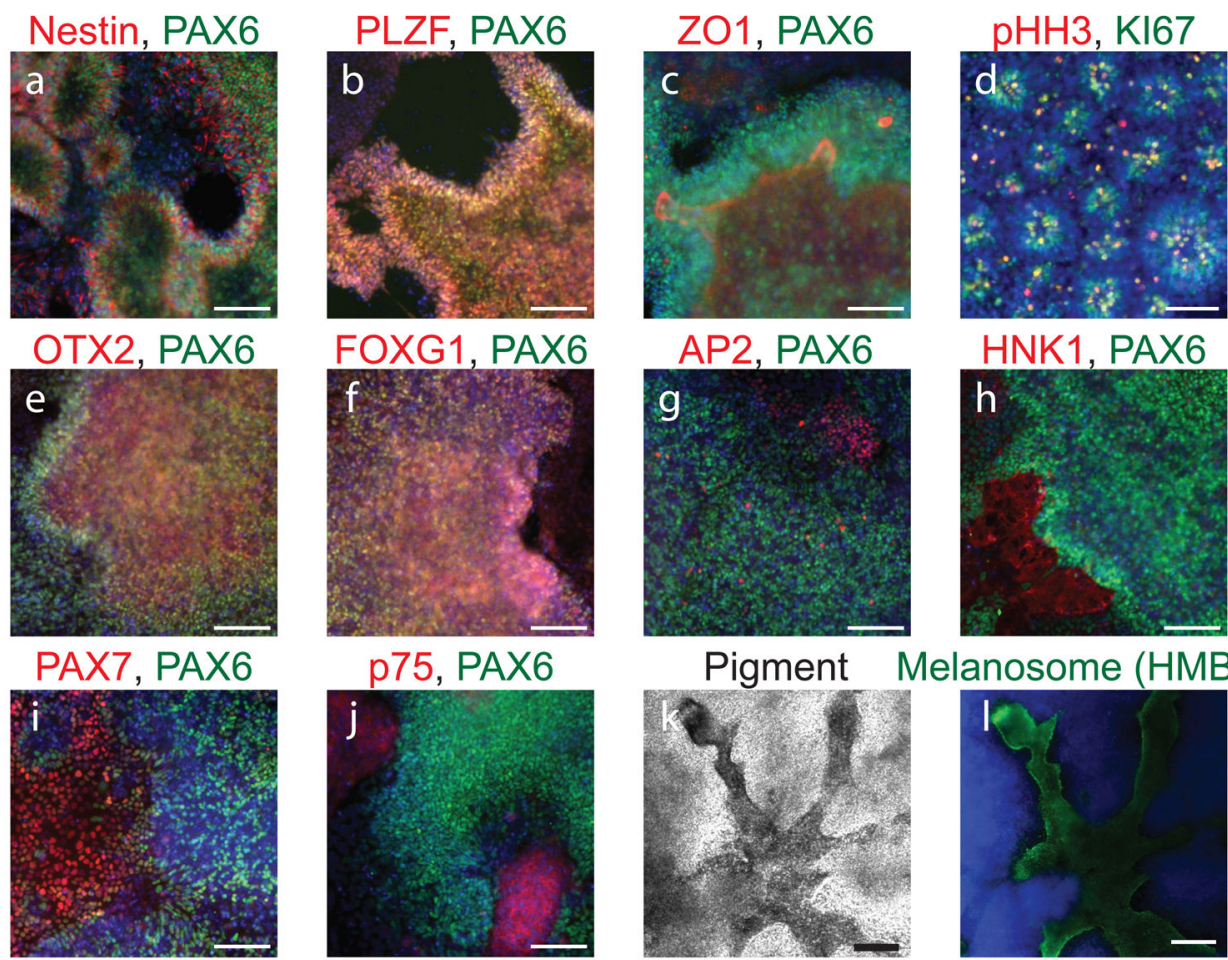

HNK1, PAX6

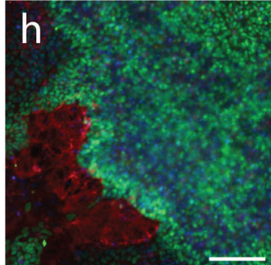

Pigment

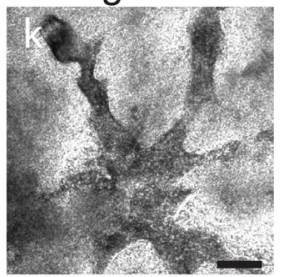

Melanosome (HMB45)

$\mathrm{m}$

TH Neuron:
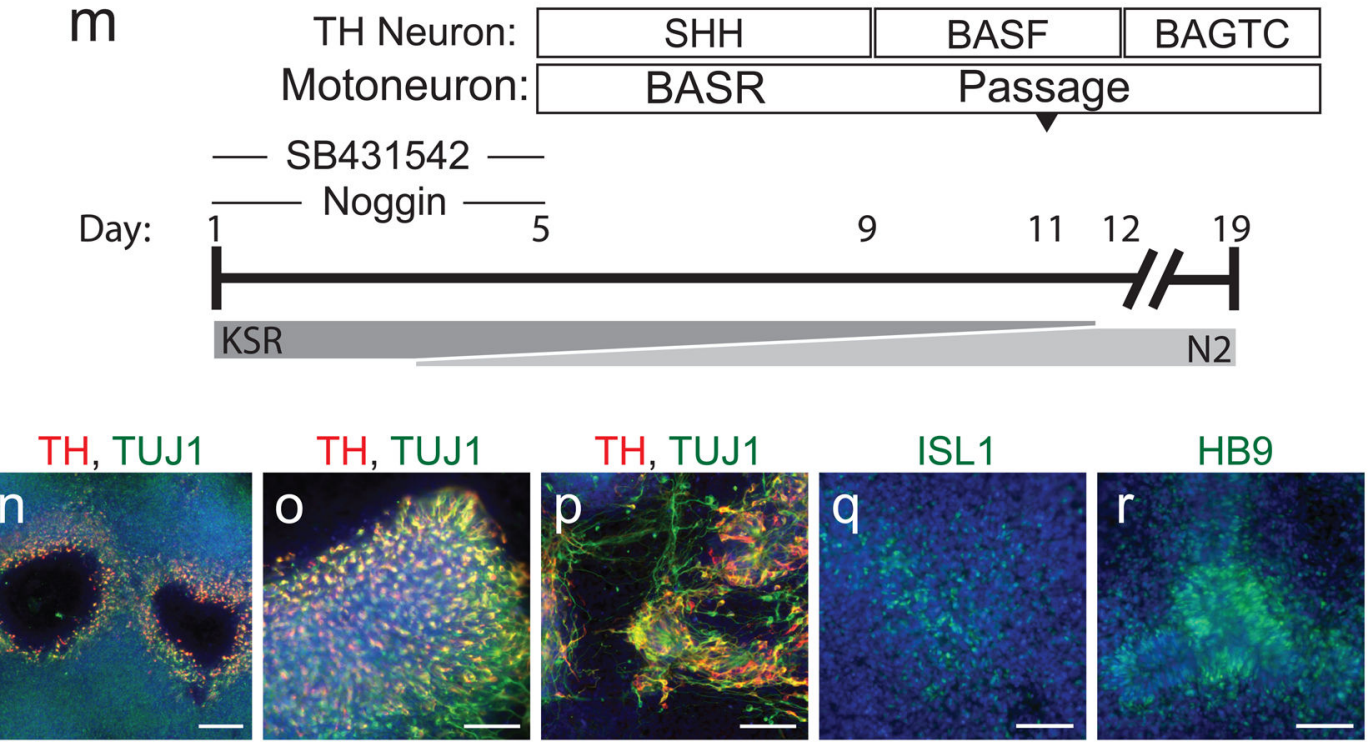

Figure 2.

Neuralization of hESC by dual SMAD inhibition permits a pre-rosette, neural stem cell with dopaminergic and motoneuronal potential. The PAX6 positive neural tissue (green) expressed rosette markers (red) (a) Nestin, (b) PLZF, (c) ZO1. (d) Rosettes are formed when $\mathrm{PAX}^{+}$tissue is passaged to conditions promoting rosettes (BASF) confirmed by KI67 (green) and luminal phospho-Histone $\mathrm{H} 3$ (red) expression, evidence of interkinetic nuclear migration. In the absence of factors that confer regional neuronal specificity, the PAX6 ${ }^{+}$ neural tissue (green) expressed (e) OTX2, and (f) BF1, indicating that the tissue defaults to 
fore-brain specification. Neural crest could be identified on the periphery of the PAX6 positive tissue (green) based on (g) AP2, (h) HNK1, (i) PAX7, and (j) p75 expression (red). Upon passage, the neural crest cells gave rise to $(\mathrm{k})$ pigmented cells (l) that expressed HMB45 (green), indicating melanosome synthesis. (m) Dopaminergic neuronal patterning was initiated with the addition of super sonic on day 5-9, followed by the addition of BDNF, ascorbic acid, sonic hedgehog, and FGF8 on day 9-12. Dopaminergic cells were maturated on days 12-19 with BDNF, ascorbic acid, GDNF, TGFb3, and cAMP.

Motoneuronal patterning was initiated at day 5 with the addition of BDNF, ascorbic acid, sonic hedgehog, and retinoic acid. Cells were passaged on day 11. (n-p) Without passage, tyrosine hydroxylase (TH) positive cells could be observed by day 19. (p) When passaged en bloc on day 12, more mature processes from TH positive cells were observed. For motoneuron induction, nuclear expression of the motoneuron markers (q) ISL1 and (r) HB9 were observed within a total of 19 days of differentiation from hESC. Scale bars: (a,b,c,e,f,g,h,i,j,o,p,q,r) - $100 \mu \mathrm{m} ;(\mathrm{c}, \mathrm{d})-50 \mu \mathrm{m} ;(\mathrm{k}, \mathrm{l}, \mathrm{n})-200 \mu \mathrm{m}$. 


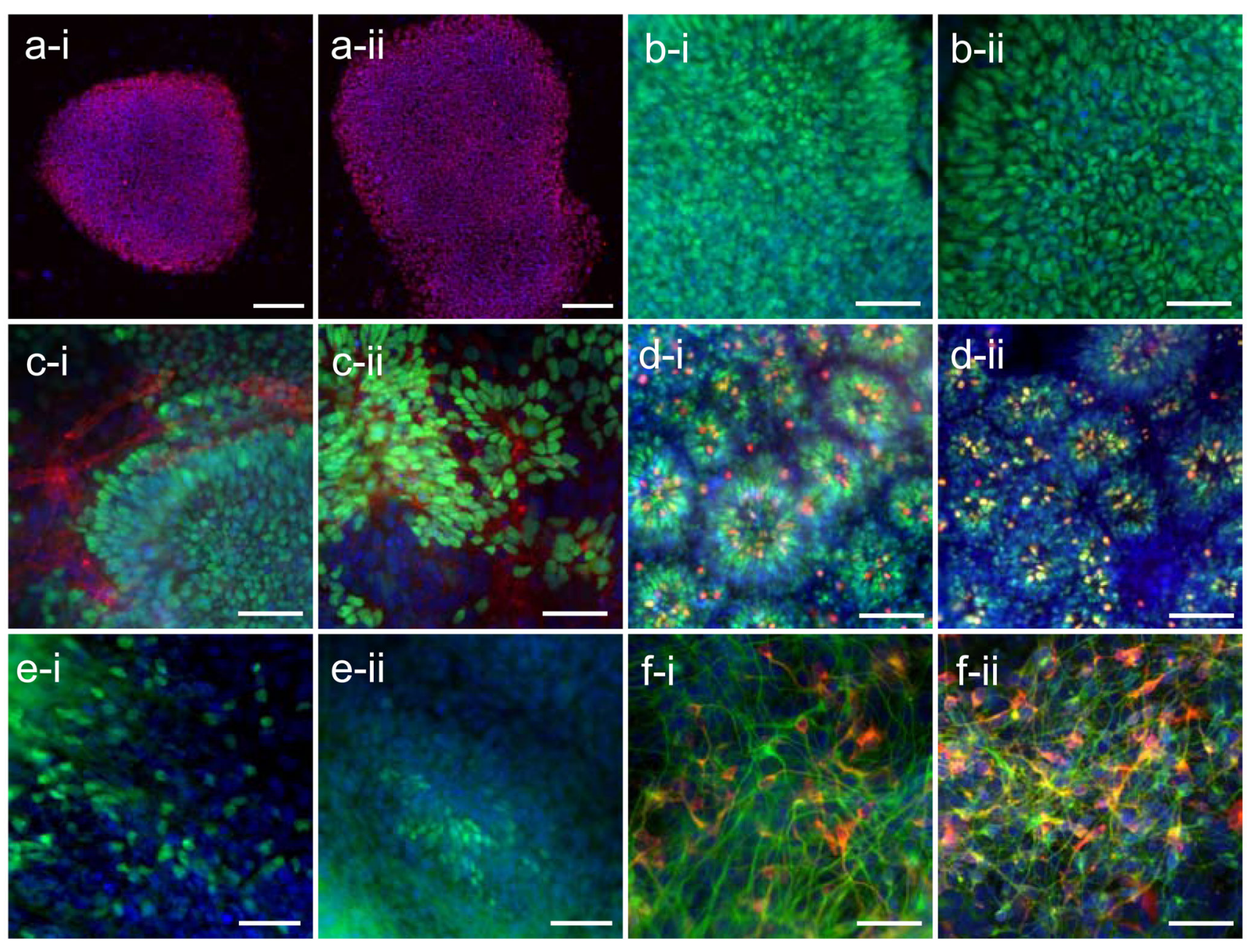

Figure 3.

Induced pluripotent stem cells (IPS) can be differentiated to neural tissue using dual SMAD inhibition and are patternable to dopaminergic neurons and motoneurons. (a-i, ii) Two IPS clones (IPS ${ }^{\mathrm{C} 14}$, IPS ${ }^{\mathrm{C} 27}$ ) were generated and screened for OCT4 (red) as well as additional pluripotency factors (Tra-1-81, Tra-1-60, SSEA-4 and Nanog, data not shown). (b-i,ii) the two clones were neuralized by dual SMAD inhibition (PAX6 expression, green), and neural crest could be observed by HNK1 staining (c-i,ii). Neural tissue from the IPS clones could be induced to form rosette-NSCs (d-i,ii) based on KI-67 (red) and phospho-histone H3 (green) expression, motoneurons (e-i,ii) based on HB9 expression (green), and dopaminergic neurons (f-i,ii) based on TUJ1 (green) and TH (red) co-expression. Scale bars: $200 \mu \mathrm{m}-(\mathrm{a})$; $50 \mu \mathrm{m}-(\mathrm{b}, \mathrm{c}, \mathrm{d}, \mathrm{e}, \mathrm{f})$. 